\title{
Conservation of temporal information by perceptual systems*
}

\author{
ROBERT EFRON† \\ Neurophysiology-Biophysics Research Laboratory \\ Veterans Administration Hospital, 150 Muir Road, Martinez, California 94553
}

\begin{abstract}
Experiments in the auditory, visual, and vibratory modality employed two brief discriminably different stimuli, which were presented with temporal asynchronies unequivocally below the Hirsh-Sherrick threshold for performing temporal order judgments. A pair of such stimuli, experienced as a unitary perceptual event, is referred to as a "micropattern" composed of two "stimulus elements." Ss could readily distinguish between two such micropatterns in which the temporal order of the stimulus elements was reversed. The discrimination was based on the perceptual dominance of the second stimulus element of each micropattern. This perceptual dominance was studied as a function of stimulus-element onset and offset asynchrony, the duration and intensity of the stimulus elements, and the difference (in frequency or wavelength) between the stimulus elements of a micropattern. The results suggest the existence of an operation of all perceptual systems in the time domain that acts to conserve information concerning the temporal order of the two stimulus elements at the expense of discriminatory acuity of the first element.
\end{abstract}

Despite the striking anatomical and physiological differences found in the various perceptual systems (with respect to the structure and function of the specialized receptor organs as well as in their central anatomical connections), all perceptual systems, nevertheless, perform certain identical operations on the incoming data. The phenomena of temporal summation, masking, and persistence (Efron, 1973) are examples of such operations in the time domain. The experiments to be described in the present report reveal the existence of another operation common to all perceptual systems in the temporal sphere-one which conserves information concerning the temporal order of two rapidly successive stimuli.

The experiments to be reported are concerned with the perceptual effects produced by two discriminably different, successive, brief stimuli when they are presented within a time span that is below the threshold at which temporal order judgments can be correctly performed. The threshold for correct judgments of the temporal order of two successive stimuli presented in the same sensory modality has been studied intensively in the past decade in trained and untrained normal, as well as in brain-damaged, Ss (Hirsh, 1959; Broadbent \& Ladefoged, 1959; Hirsh \& Sherrick, 1961; Efron, 1963; Hirsh \& Fraisse, 1964; Edwards \& Auger, 1965; Holmes, 1965; Lowe \& Campbell, 1965; Homick, Elfner, \& Bothe, 1969; Jerger et al, 1969; Gengel \& Hirsh, 1970; Swisher \& Hirsh, 1972). In these various experiments, the two stimuli to be temporally ordered were presented either to different groups of receptors (in which case they could be physically identical) or to the same receptors (in which case they must be discriminably different). In either case, Hirsh and Sherrick (1961) found in trained psychophysical Ss that temporal order

\footnotetext{
*From the Veterans Administration Hospital and the Department of Neurology, University of California, School of Medicine, Davis, California.

$\dagger$ I would like to thank M. Corder and M. Strachan for being patient and good-humored Ss for these tedious experiments.
}

judgments could no longer be made correctly more than $75 \%$ of the time when the interval between the onsets of two stimuli (stimulus onset asynchrony-SOA) was reduced to $20 \mathrm{msec}$. The same value was found for the auditory, visual, and tactile modality and also for cross-modality temporal order judgments. Corresponding values of approximately $60 \mathrm{msec}$ were obtained by Efron (1963) and Hirsh and Fraisse (1964) in untrained normal Ss, and values of 150 to $600 \mathrm{msec}$ have been obtained with adult patients suffering from lesions of the dominant temporal lobe by Efron (1963), Edwards and Auger (1965), Holmes (1966), and Swisher and Hirsh (1972).

It should be noted that in the works cited, the stimulus parameters, the psychophysical methods, and the criteria used by the Ss varied widely. The striking effect of training and criteria on the measured thresholds for temporal order judgments in audition was reported by Broadbent and Ladefoged (1959), who found in their experiment that the threshold, which was initially over $150 \mathrm{msec}$, fell precipitously as Ss had more prolonged exposure to the task. The final thresholds were of the order of those reported by Hirsh (1959) and Homick et al (1968)-which are the lowest values found in the literature (17 and $14 \mathrm{msec}$, respectively, in the auditory modality).

The specific purpose of the present study was to determine how temporal order information is processed by the nervous system when the two stimuli are presented at stimulus onset asynchronies which are unequivocally below the threshold for temporal order judgments. If all temporal order information is not lost, then in what form is it retained?

\section{EXPERIMENTAL PARADIGM}

Two brief, discriminably different stimuli are delivered to a sense organ within such a short interval that the $\mathrm{O}$ is unable to report the correct temporal order and experiences the two stimuli as a single perceptual 


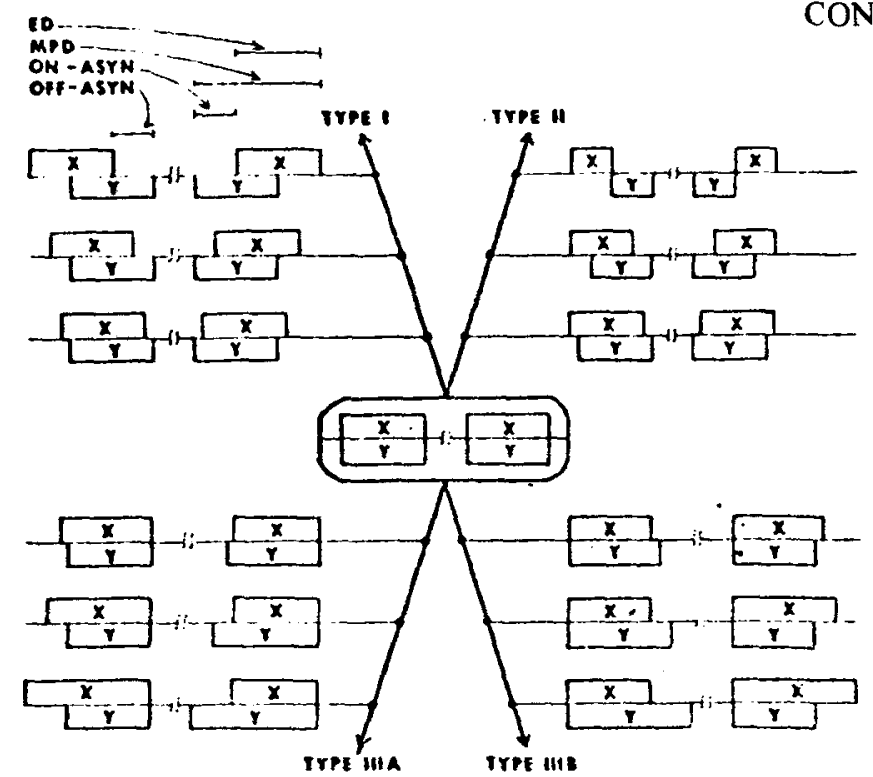

Fig. 1. Representation of the experimental paradigm. In center of figure are two identical micropatterns, each composed of Stimulus Elements $X$ and $Y$. The radial vectors illustrate four types of alterations to which the identical micropatterns were subjected. For each type of alteration, the stimulus element asynchrony increases in the direction of the vector. ED indicates the element duration; MPD, the micropattern duration; ON-ASYN, the asynchrony between the onsets of the two elements of a micropattern: and OFF-ASYN, the asynchrony between the offsets.

event. For example, the two stimuli might be a $10-\mathrm{msec}$ burst of a $1,000-\mathrm{Hz}$ signal followed immediately (stimulus onset asynchrony $=10 \mathrm{msec}$ ) by a $10-\mathrm{msec}$ burst of a $2,000-\mathrm{Hz}$ signal of the same energy). The $\mathrm{S}$ will hear a single complex sound, equivalent to a two-note chord, in which he can identify both the highand low-pitch components-but he will be unable to report (at any level above chance) the actual temporal order of the two tones. The two questions to which the following experiments are addressed are: (a) Can the $S$ distinguish between the perceptual event just described and a second event in which the same two tones are presented but in reversed temporal order, that is, when the $2,000-\mathrm{Hz}$ tone precedes the $1,000-\mathrm{Hz}$ tone? If discrimination between these two perceptual experiences is possible, then temporal order information is conserved, despite the fact that temporal order judgments cannot be made. (b) If discrimination is possible, on what perceptual feature of the stimuli is the distinction based?

Essentially identical experiments were performed in three sensory modalities-audition, vibration, and vision. In each modality, the $S$ was required to discriminate between two perceptual events, each of which was composed of two stimuli having asynchronous onsets or offsets which are below the threshold for temporal order judgments. To avoid confusion when speaking of "events" or "pairs of stimuli," the following nomenclature will be adopted: Each pair of stimuli producing a unitary perceptual event will be referred to as a "micropattern." (The term "micropattern" has been used to distinguish sharply between the temporal parameters of the pattern discrimination tasks described in this report and the temporal parameters used in the many studies of pattern discrimination in man and animals where sequences of discriminably different stimuli are employed in which the intervals between the stimuli are far above the threshold for temporal order judgments.) Each micropattern is composed of two discriminably different stimulus "elements"-X and $Y$. Expressed in these terms, the $S s$ are required to discriminate, using "same" or "different" replies, between two micropatterns (presented $0.5 \mathrm{sec}$ apart), each of which is composed of the same two elements.

The duration of a stimulus element will be referred to as "element duration" (ED). The duration of the micropattern, measured from the onset of the first element to the termination of the second element will be called the "micropattern duration" (MPD). The time relationship between Elements $X$ and $Y$ will be described in terms of their "element onset asynchrony" (ON-ASYN) and "element offset asynchrony" (OFF-ASYN). In the experiments to be described, the alterations of ED, ON-ASYN, OFF-ASYN, and MPD on micropattern discrimination were studied.

Figure 1 illustrates schematically the basic alterations of these four temporal parameters that were employed in the present experiments. In the center of the figure are two micropatterns, each composed of two discriminably different stimulus elements ( $X$ and $Y$ ). For schematic convenience, Stimulus Element $X$ is always drawn above the line and Stimulus Element $Y$ is drawn below the line. The ON-ASYN and OFF-ASYN are both zero, and the EDs and MPDs are equal. These two micropatterns are thus physically identical and perceptually indistinguishable. In each of the four types of alteration (denoted by the four radial vectors), the asynchrony between the stimulus elements is increased. In Type I alterations, the ED is held constant and the ON-ASYN and OFF-ASYN are increased equally. The MPD must necessarily increase since the EDs are of constant duration. In Type II alterations, the MPD is held constant and the ON-ASYN and OFF-ASYN are again increased equally. In this case, the EDs must necessarily decrease in duration since the MPDs are of constant duration. In Type IIIA alterations, the OFF-ASYN is always zero and only the ON-ASYN is increased. This necessarily results in an increase of the duration of one of the elements, and this will be reflected in an increase of the MPD. In Type IIIB alterations, the ON-ASYN is always zero and only the OFF-ASYN is increased. One ED and the MPD must also increase, as was the case for Type IIIA alterations.

As the element asynchrony in each type of alteration is increased, the two micropatterns must become discriminable: Type I and Type II alterations will 
ultimately result in discriminable micropatterns when the Hirsh-Sherrick threshold is exceeded. That is to say, the Ss will be able to distinguish different micropatterns by identifying the temporal order of the two elements: Type IIIA and Type IIIB alterations will also ultimately become discriminable, since one of the elements of each micropattern will have a greater subjective intensity as its duration increases in relation to the other. It is important to note that the magnitude of the element asynchronies employed in the present experiments were always sufficiently small so that the micropatterns used could never be discriminated on the basis of perceived temporal order or perceived intensity differences.

For any set of values of ED, ON-ASYN, OFF-ASYN, and MPD illustrated in Fig. 1, four modes of presentation of the two micropatterns are possible and all were employed in random presentation. In Fig. 2, the four modes of presentation are illustrated only for the two micropatterns in the upper left corner of Fig. 1.

The nontemporal parameters of the micropatterns-i.e., the parameters which made Elements $X$ and $Y$ discriminably different-were the difference in frequency $(\Delta f)$ between the two elements (in the auditory and vibratory experiments) and the difference in wavelength $(\Delta \lambda)$ between the two stimulus elements (in the visual experiments). The effect of variation of $\Delta f$ and $\Delta \lambda$ on micropattern discrimination was studied for selected values of the temporal parameters (ED, ON-ASYN, OFF-ASYN, and MPD).

\section{METHODS}

\section{Psychophysical}

The method of forced choice ("same"-"different") was used for all determinations of Experiments I, II, IIIA, and IIIB. For each data point, 100 trials were used, 25 of each of the four possible modes of presentation illustrated in Fig. 2, which were presented in pseudorandom order. These 100 trials were completed in a single session. The percentage of correct replies was calculated for each 100 trials. (A method of adjustment was used for Experiments IV and $\mathrm{V}$ and will be described later.)

The two micropatterns (separated by an interval of $0.5 \mathrm{sec}$ ) were presented every $3 \mathrm{sec}$, and a verbal response was required within that interval. In the visual experiments, the use of a dental bite for head fixation precluded verbal responses and a hand signal was substituted.

Two highly trained psychophysical Ss were used for all experiments. With one exception, all of the experiments were completed by the same two Ss. The exception was the single experiment in the vibratory modality which could not be performed by S M.C., who had had no previous experience with vibratory stimuli. Another $S$ (the author) was used for this experiment.

For each experimental session (which consisted of a single set of stimulus parameters), a brief training period was given. The Ss were presented with two trial exposures of each of the four possible modes of presentation of the two micropatterns (see Fig. 2). Prior to each of these eight training trials, the $S$ was given advance knowledge of the correct reply. The $\mathrm{S}$ was not told the temporal order of Elements $X$ and $Y$; he was informed only that the two micropatterns would be same or different. Following the eight training exposures, which were intended to establish the criterion of judgment, the 100 test trials were
IS MICROPATTERN
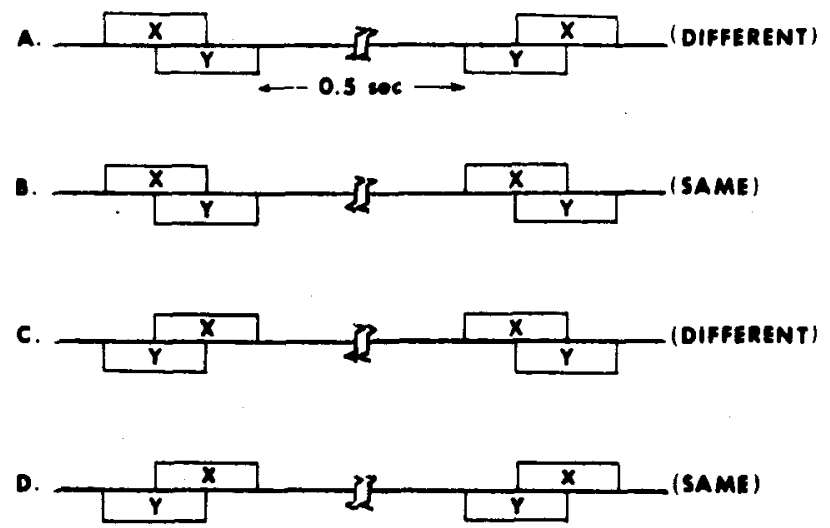

Fig 2. Representation of the four possible modes of presenting two micropatterns composed of identical stimulus elements, $X$ and $Y$.

presented with no feedback. For every change in stimulus parameters, the Ss were again given eight training trials with preknowledge of the correct reply, followed by 100 trials without feedback.

\section{Instrumental}

Element duration (ED), element onset asynchrony (ON-ASYN), element offset asynchrony (OFF-ASYN), micropattern duration (MPD), the 0.5-sec interval between micropatterns, and the 3.0-sec interval between trials were controlled with a digital logic system (Iconix 6257-6010-6171), which was used for all experiments. Its accuracy was $\pm .01 \mathrm{msec}$.

\section{Auditory Experiments}

The audio signals were generated by two independent continuous sine-wave generators, the frequency of which was always monitored by separate counter timers. These signals were passed through two audio gates (Iconix 0137), which shaped the rise and decay times of the stimulus elements. An exponential rise and decay time of approximately $7 \mathrm{msec}$ was used $(1 / \mathrm{e}=$ $5.0 \mathrm{msec}$ ). The timing of gate opening and closing was controlled by the Iconix digital logic system, which was not synchronized to the frequency of either of the two sine-wave generators. The output from each audio gate was passed through an attenuator (Hewlett-Packard 350D), electrically added (Philbrick P-85AU), passed through a filter (Krohnhite $3500-\mathrm{R}$ ), with a bandpass of $1,000-2,000 \mathrm{~Hz}$, and finally sent to the $S$ 's right ear via headphones (Koss-Pro 4A) in an audiometric testing chamber (IAC 401-A). For the experiments of Type V (to be described later), which were performed only in the auditory modality, an additional sine-wave generator was placed in the testing chamber. The output of this third sine-wave generator, which was adjusted by the $S$, was passed through a third audio gate and mixed with the outputs of the other two oscillators. The third oscillator produced only one of the elements in the second micropattern (denoted by the symbol V in Fig. 6).

The steady-state amplitude of each stimulus element was $1.0 \mathrm{~V}$ peak-to-peak, measured across the coil of the earphone. This voltage resulted in a sound level of $80 \pm 0.5 \mathrm{~dB}$ (re .0002 dynes $/ \mathrm{cm}^{2}$ ), measured at the surface of the earphones with a 6-cc coupler (Bruel \& Kjaer, Type 2603 amplifier, Type 4134 microphone). The frequencies used were between 1,300 and $1,940 \mathrm{~Hz}$-the essentially flat portion of the auditory sensitivity curve. In those experiments, in which stimulus frequencies were 


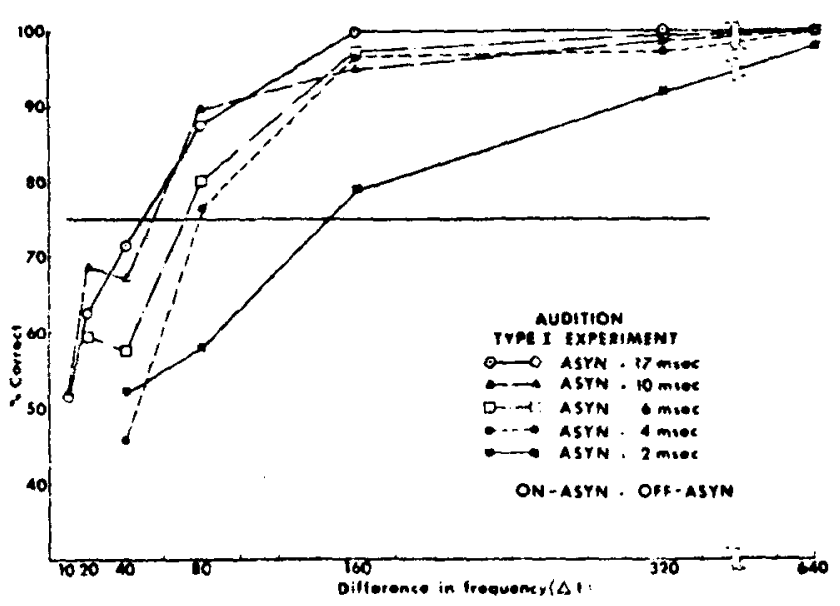

Fig. 3. Discrimination of micropattems, Experiment I (audition). For all micropatterns, the ON-ASYN was equal to the OFF-ASYN (see Type I alterations in Fig. 1). Each set of symbols $(\odot, \Delta, \boxminus, \bullet, \varpi)$ represents a different element asynchrony. Data of two Ss.

outside this narrow range, the bandwidth of the filter was suitably increased.

\section{Vibratory Experiments}

The identical apparatus was used, except that the output of the filter (bandwidth of $50-500 \mathrm{~Hz}$ ) was fed to a power amplifier (Ling Model 25), which operated a vibrator (Ling Model 102). The rise and decay times of the stimulus elements was $10 \mathrm{msec}$. A probe, attached to the vibrator, was applied to the tip of the right third finger. The skin deformation was $0.04 \mathrm{in}$. peak-to-peak (see Efron, 1973, for details). The frequencies of vibration were between 100 and $400 \mathrm{~Hz}$.

\section{Visual Experiments}

The visual stimuli were derived from two glow modulators (Sylvania $\mathrm{R} 1131 \mathrm{C}$ ), driven by the Iconix digital logic system. The light output from each glow modulator was passed through neutral density filters and then narrow-band $(9-\mathrm{m} \mu$ half bandwidth) interference filters (Bausch \& Lomb). The two monochromatic light beams were combined, using a prism. and fell upon a disk of ground glass. The ground glass then served as a secondary source (of mixed light) which was focused on the S's cornea for a Maxwellian view. In other experiments, wide-band Wratten colored filters were used. The $S$ 's head was fixed by use of a dental bite, and he observed the visual stimuli through an aperture placed on the surface of the lens used to focus the light on the cornea. The visual target was $1 \mathrm{deg} 45 \mathrm{~min}$ of visual angle in diam and had a luminance of approximately $15 \mathrm{fL}$. The $S$ viewed this target in a darkened, but not lightproof, room. Sufficient ambient light was available to enable the $S$, after a period of dark adaptation, to see the aperture without a fixation light.

\section{RESULTS}

\section{Audition}

\section{Experiment I}

Figure 3 presents the data obtained from a series of experiments of Type I (Fig. 1, upper left group of schematics), where the ON-ASYN was always equal to the OFF-ASYN. Since the data of both Ss showed no significant differences, the results were collapsed across Ss. The ordinate is the percentage of correct replies (50\% being the chance level); the abscissa is the difference in frequency of the two elements.

For the experiments reported in Fig. 3, five values of element asynchrony $(2,4,6,10$, and $17 \mathrm{msec})$ were used, and the results for each value of asynchrony are denoted by a common symbol. The element durations were $17 \mathrm{msec}$ (including the $7-\mathrm{msec}$ rise and decay times). Since the EDs were of equal duration, the MPD for each of the above five values of element asynchrony were $19,21,23,27$, and $34 \mathrm{msec}$, respectively. The sound levels of the two elements were equal $(80 \mathrm{~dB}$ SPL). The frequency of Element $X$ was always $1,300 \mathrm{~Hz}$; Element $Y$ was $1,320,1,340,1,380,1,460,1,620$, or $1,940 \mathrm{~Hz}$ in six different experiments. These values give rise to frequency differences $(\Delta f)$ of $20,40,80,160$, 320 , and $640 \mathrm{~Hz}$, which are noted on the abscissa. Each point in the graph is obtained from 200 trials (25 of each of the four combinations illustrated in Fig. 2 for each of the two Ss). A horizontal line, drawn at the $75 \%$ level, defines the threshold value used in these experiments.

As can be seen in Fig. 3, the Ss were always above threshold for discriminating differences between the first and second micropatterns when the difference in frequency between the two elements and the element asynchrony was large, but fell below threshold when the difference in frequency between the two elements was made smaller and when the element asynchrony was reduced. A chance level of performance was found with the smallest frequency differences and shortest element asynchronies. For any specific $\Delta f$, the Ss generally had higher discrimination performances for large-element asynchronies, and their performance deteriorated as the element asynchrony was decreased.

From these results, it was quite obvious that the Ss could distinguish the difference between Micropatterns $X Y$ and $Y X$, even with small differences in frequency between Elements $X$ and $Y$. With larger differences in frequency, the $S$ s remained above threshold, even with element asynchronies of $2 \mathrm{msec}-\mathrm{a}$ value which is far below the Hirsh threshold of $17 \mathrm{msec}$ for perceived temporal order judgments in audition. In additional Type I experiments (not illustrated), the same two Ss were still always above threshold, with an ED of $12 \mathrm{msec}$, an element asynchrony of $2 \mathrm{msec}$, a MPD of $14 \mathrm{msec}$, and a $\Delta f$ of $5,000 \mathrm{~Hz}$. Informal studies on 10 other Ss revealed the same phenomena. From these results, it was concluded that temporal order information is conserved, even at relatively short-element asynchronies-despite the fact that temporal order judgments could not be made. On what perceptual cue, then, was the micropattern discrimination performed? 
All Ss observed that they were basing their reports ("same" or "different") on an obvious difference in the dominant pitch of the two micropatterns. For example, if the Ss were asked to compare a micropattern in which the sequence was $1,300-1,940 \mathrm{~Hz}$ with a second micropattern in which the sequence was $1,940-1,300 \mathrm{~Hz}$, they all reported that the first micropattern had a higher dominant pitch than the second. The Ss' replies thus correlated perfectly with the temporal order of the stimulus elements: The stimulus element in a micropattern which was presented last dominated the perceptual experience of that micropattern.

It should be stressed that the Ss had no knowledge of the temporal order of the elements or that their perceptual experience of each micropattern was correlated with the temporal order of its elements. When they were ultimately informed of the correlation (at the end of the series of experiments), the Ss could then infer the temporal order of the stimulus elements. However, during the course of the experiments, this information was not available to them and this inference could not have been made.

In addition to the use of micropatterns in which the elements were tone bursts of two different frequencies, Experiment I was also performed using micropatterns consisting of a tone and a white noise burst and micropatterns consisting of two pink noise bursts (of different bandwidths). Again, the Ss reported that they made their discriminations on a readily perceptible difference in the dominant pitch of the two micropatterns--the spectral characteristics of the second element always dominating the pitch of the micropattern.

\section{Experiment II}

Figure 4 presents the pooled data of both Ss from a series of Type II alterations (Fig. 1, upper right schematics), where the ON-ASYN was always equal to the OFF-ASYN. The ordinate is the percentage of correct replies; the abscissa is the difference in frequency between the two elements. The same values for the frequency of the elements was employed as in Experiment I, and the same values of element asynchrony $(2,4,6,10,17 \mathrm{msec})$ were also repeated. In all of these experiments, the MPD was held constant at $34 \mathrm{msec}$. Since the MPDs were of constant duration for each of the above five values of element asynchrony, the EDs were $32,30,28,24$, and $17 \mathrm{msec}$, respectively. The sound levels of the two elements were equal $(80 \mathrm{~dB}$ SPL). The top curve of Fig. 4 obtained with an ASYN of $17 \mathrm{msec}$ was identical with the stimulus parameters of the top curve of Fig. 3 (ASYN = 17) and is merely the same experiment repeated several months later.

A comparison of Fig. 4 with Fig. 3 reveals that Type II alterations resulted in a deterioration of discriminatory performance for element asynchronies of 2,4 , and $6 \mathrm{msec}$. The explanation of this result will be

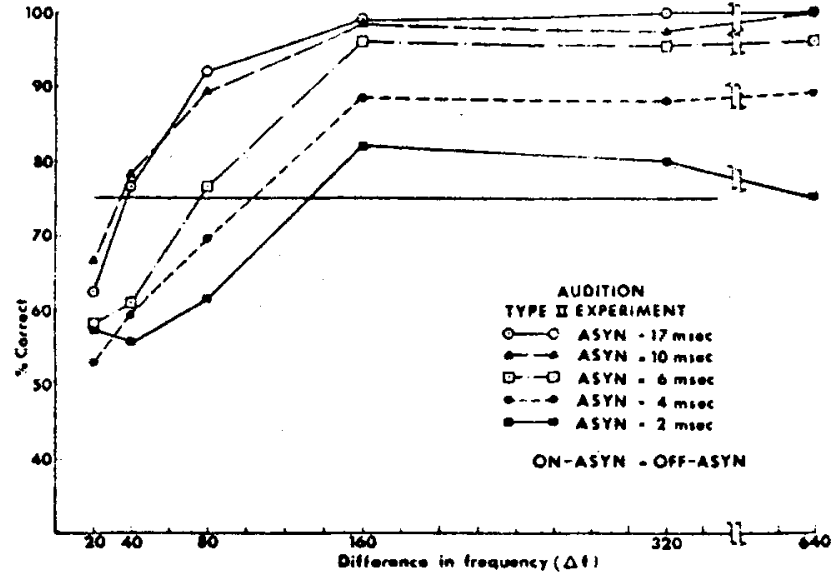

Fig. 4. Discrimination of micropatterns, Experiment II (audition). For all micropatterns, the ON-ASYN was equal to the OFF-ASYN (see Type II alterations in Fig. 1). Each set of symbols $(\odot, \Delta, \boxminus, \bullet, \square)$ represents a different element asynchrony. Data of two Ss. Curves marked $\odot-\odot$ in this figure are repeat studies of similarly marked curves in Fig. 3.

deferred until after the results of Experiments IIIA and IIIB are presented.

\section{Experiments IIIA and IIIB}

In Type I and Type II alterations, the micropatterns had equal element onset and offset asynchronies. In Type III alterations, however, the effects of the element onset and offset asynchrony were studied separately. In Experiment IIIA, the two elements (of both micropatterns) terminate synchronously, but commence asynchronously (see Fig. 1, lower left schematics). In Experiment IIIB, the two elements commence synchronously, but terminate asynchronously (see Fig. 1, lower right schematics).

The results of some of these experiments are listed in Table 1. The upper half of this table contains the results of five IIIA experiments with different stimulus parameters. The lower half of the table contains the parallel results obtained in each of the five IIIB experiments with identical parameters. In all 10 experiments, the two elements were tone bursts of 1,300 and $1,500 \mathrm{~Hz}$ of equal amplitude $(\Delta f=200)$. The data has been collapsed across Ss as there were no significant differences.

The results (Column 1) indicate that the two micropatterns cannot be discriminated when the element asynchrony of $2 \mathrm{msec}$ is at the onset or leading end of the micropattern (the values are below $75 \%$ threshold level for Experiment IIIA), but are almost perfectly discriminated when there is a $2-\mathrm{msec}$ offset asynchrony (Experiment IIIB).

Despite the fact that the same amount of temporal order information $(2.0 \mathrm{msec})$ is provided by the element onset asynchrony (Experiment IIIA) as is provided by the element offset asynchrony (Experiment IIIB), the 
Table 1

\begin{tabular}{lccccc} 
& Column 1 & Column 2 & Column 3 & Column 4 & Column 5 \\
\hline Element Durations & $17-19 \mathrm{msec}$ & $27-29 \mathrm{msec}$ & $57-59 \mathrm{msec}$ & $57-67 \mathrm{msec}$ & $17-27 \mathrm{msec}$ \\
Element Asynchrony & $2.0 \mathrm{msec}$ & $2.0 \mathrm{msec}$ & $2.0 \mathrm{msec}$ & $10.0 \mathrm{msec}$ & $10.0 \mathrm{msec}$ \\
Element Onsets Asy nchronous (Experiment IIIA) & $61 \%$ & $43 \%$ & $51 \%$ & $57 \%$ & $80 \%$ \\
Element Offsets Asynchronous (Experiment IIIB) & $96 \%$ & $85 \%$ & $65 \%$ & $100 \%$ & $100 \%$ \\
\hline
\end{tabular}

perceptual system appears to make very little, if any, use of the former.

In the Type III alterations (unlike those of Types I and II), the energy in each element was unequal. In the first pair of Type III alterations (Column 1), the element durations were 17 and $19 \mathrm{msec}$ (including rise and decay times). The longer element would be expected (by virtue of temporal summation) to sound louder than the shorter element, and its pitch would be expected to dominate the perception of that micropattern. For example, in the trials in which the $1,500-\mathrm{Hz}$ element was $19 \mathrm{msec}$ and the $1,300 \cdot \mathrm{Hz}$ element was $17 \mathrm{msec}$, the $1,500-\mathrm{Hz}$ element would be expected to be slightly louder. When this micropattern was compared to one in which the $1,300-\mathrm{Hz}$ element was $19 \mathrm{msec}$ and the $1,500-\mathrm{Hz}$ element was $17 \mathrm{msec}$, the small difference in loudness of the different elements might make the two micropatterns discriminably different. However, this was not the case. In the IIIA experiments of Column 1, this differential loudness factor was apparently not of sufficient magnitude to be used as a discrimination cue. since the Ss' performance was near the chance level. It can be concluded, therefore, that the high level of discriminatory performance in the parallel IIIB experiment (Column 1) could not be attributed to a differential loudness factor, but must be attributed to the element offset asynchrony per se. The results of Experiment III thus indicate that the ON-ASYN of the micropatterns of Experiment I played little or no role in improving the discrimination performance.

The second and third columns of Table 1 show the consequences of increasing the duration of both elements while keeping the ON-ASYN and OFF-ASYN at $2.0 \mathrm{msec}$. Again, Experiment IIIB yields a better discriminatory performance than the equivalent Experiment IIIA. However, it is also quite clear that increasing the duration of the elements while maintaining a 2-msec offset asynchrony deteriorates the performance in the IIIB experiment (Columns 2 and 3 ). This is exactly what occurred in Experiment II as compared to Experiment I (compare Fig. 4 with Fig. 3), where performance deteriorated when the element durations were made longer (in Experiment II).

When the element durations are made longer in both Experiment II and Experiment III, the 2-msec OFF-ASYN represents a smaller proportion of the overall micropattern duration. This decreased ratio could account for the decreased performance. In the fourth pair of experiments (Table 1, Column 4), the element durations were 57 and $67 \mathrm{msec}$ (i.e., with a $10 \mathrm{msec}$ asynchrony), reproducing the ratio used in the experiments of Column 1. Both Ss obtained a 100\% level of discrimination in Experiment IIIB, but were still at chance level of performance for Experiment IIIA. From the pattern of results in the first four pairs of these experiments, it appears that the trailing end of the micropattern becomes a less effective discrimination cue as the element durations are prolonged. The effectiveness can be restored, however, with an increase of the element offset asynchrony.

The first four pairs of these experiments indicate that the pertinent information used by the $S$ to discriminate micropatterns lies at the end of the pattern (the element offset asynchrony), and that the element onset asynchrony provides little, if any, information. However, a very large element onset asynchrony will also improve discriminatory performance. In the fifth pair of experiments (Column 5), element durations of 17 and $27 \mathrm{msec}$ were used. In this case, the Ss achieved a higher level of performance in Experiment IIIA than was obtained in Experiment IIIA of Column 1. The improved performance in this last experiment (Column 5) can be easily accounted for by the fact that the longer element had nearly twice the energy of the shorter element. With twice the energy, the longer element would be expected to have a discriminable effect on the dominant pitch of the micropattern (by virtue of its increased loudness) and the micropattern discriminating performance should be improved. Despite this improvement, the Ss achieved only an $80 \%$ correct discrimination in Experiment IIIA compared to a $100 \%$ performance in the equivalent Experiment IIIB (Column 5)-indicating once again that the element offset asynchrony provides the more salient cue for micropattern discrimination.

It should be noted that, while Hirsh's (1959) experiment on temporal order judgments employed the paradigm of Type IIIA alterations (see Fig. 1), he employed element durations of $500 \mathrm{msec}$. His Ss were at threshold with an ON-ASYN of 17-20 msec. With such a large ratio of ED to ON-ASYN, the dominance effect described in the present report is not observed, and would not have interfered with his results or conclusions (as suggested by Broadbent and Ladefoged). It would appear that the ON-ASYNs (when of sufficient magnitude) will enable the Ss to make temporal order judgments, but do not give rise to the perceptual dominance effect.

It is difficult to explain the failure of the element onset asynchrony (in the IIIA experiments) to give rise to any perceptual dominance of the leading (longer) element or to any other perceptual cues which 
facilitated discrimination between micropatterns. A possible explanation may be a delay in an attention switching mechanism suggested by Kristofferson $(1967 \mathrm{a}, \mathrm{b})$ or a delay in a channel switching mechanism postulated by Broadbent (1957). By the time the nervous system has "switched" to the micropattern, the salient information (in a 2-msec ON-ASYN) may be lost. To test this possibility, each micropattern was preceded by a click-the intended purpose of which was to "switch" or "cue" the auditory system just before the micropattern was delivered. Despite a wide range of click intensities and time relationships to the micropattern, no improvement in discriminatory performance was noted in experiments of the IIIA paradigm by the introduction of a click. This negative finding does not, of course, completely exclude a channel switching explanation, since the "correct" parameters of the switching stimulus may not have been employed. It does, however, undercut this explanation.

\section{Experiment IV}

In Experiments I, II, and III, the Ss indicated that they based their replies on the dominant pitch of the micropatterns. The second, or trailing, element of each micropattern dominated the perceptual experience of that micropattern. The object of Experiment IV was an attempt to reduce the dominating perceptual effect of the second element of a micropattern by a reduction of its energy and to increase the perceptual effect of the first element by an increase of its energy-the total energy in the micropattern to be unchanged.

The $\mathrm{S}$ was presented with two micropatterns. The first, or "standard" micropattern, consisted of the element sequence $X_{s}-Y_{s}$ (where the subscript denotes that these elements are part of the standard micropattern). $X_{\mathrm{s}}$ was $1,500 \mathrm{~Hz}$ and $Y_{\mathbf{s}}$ was $1,300 \mathrm{~Hz}$, and their amplitudes and durations were equal-the former being $80 \mathrm{~dB} \mathrm{SPL}$ and the latter being $12.0 \mathrm{msec}$ (including the $7.0-\mathrm{msec}$ rise and decay time). The second (or "test") micropattern (presented $0.5 \mathrm{sec}$ later) always consisted of the reverse temporal sequence $\left(Y_{t}-X_{t}\right)$, where $Y_{t}$ was $1,300 \mathrm{~Hz}$ and $X_{t}$ was $1,500 \mathrm{~Hz}$.

This paradigm is illustrated by the two micropatterns of Fig. 5A. It will be noted that in Fig. 5, which represents the temporal parameters of this auditory experiment, the rise and decay times of each element are illustrated. When presented with these two micropatterns, the $S$ could discriminate a difference at the $100 \%$ level of performance. Using a method of adjustment, he was then required to alter the ratio of energies of the two elements of the test micropattern (by adjusting their relative durations) until he could no longer distinguish this micropattern from the standard micropattern (which was never altered). The $\mathrm{S}$ was permitted to examine as many pairs of micropatterns as he wished until he was satisfied with his adjustment. At the completion of this task, the values of the durations

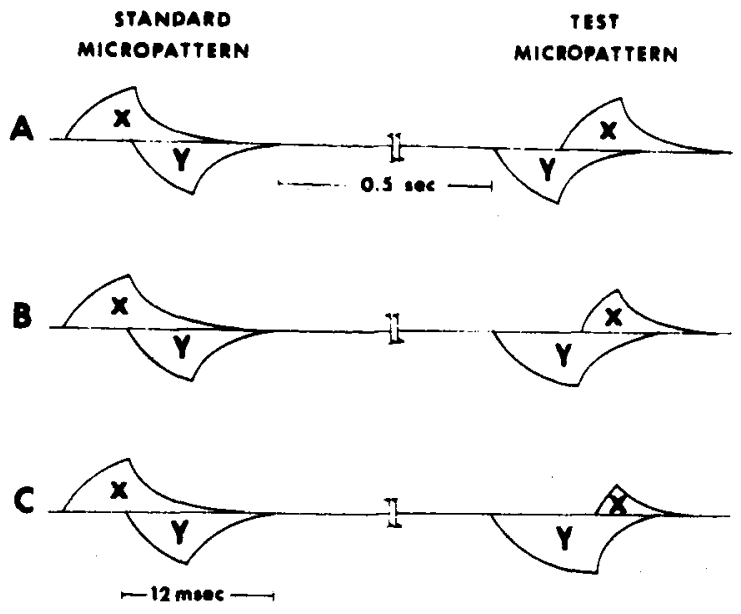

Fig. 5. Paradigm of Experiment IV (audition). The $S$ is always presented with two micropatterns in which the temporal order of the elements is reversed. Line $A$ represents the initial values set by $E$. Line B represents one step in the adjustment by the $S$ of the test micropattern. Line $C$ represents the final step of the S's adjustment of the test micropattern (see Table 2 for results).

of $Y_{t}$ and $X_{t}$ were recorded by the $E$. The durations of $Y_{t}$ and $X_{t}$ were again made equal by the $E$, and the $S$ repeated the adjustment. This adjustment procedure was repeated 30 times. Two stages in the adjustment procedure which altered the relative durations of $Y_{t}$ and $X_{t}$ in the test micropattern are illustrated in Fig. 5, B and $\mathrm{C}$.

The $S$ altered the durations of $Y_{t}$ and $X_{t}$ in $0.1-\mathrm{msec}$ steps by turning a set of switches. The equipment was programmed so that any increase in the duration of $Y_{t}$ was exactly counterbalanced by a reduction in the duration of $X_{t}$. The sum of the durations of the two elements in the test micropattern was thus constant and always equal to the sum of the durations of the two elements in the standard micropattern.

Table 2 contains the results of this experiment. At the null point of the Ss' adjustment, the duration of the first element of the test micropattern was $15.25 \mathrm{msec}$; the second element was $8.75 \mathrm{msec}$. At these settings, the Ss found the test and standard micropattern to be indiscriminable.

The results of the experiment thus indicate that the dominating effect of the second element of the test micropattern could be reversed by a reduction in its duration (energy) coupled with an increase in the duration (energy) of the first element.

When the Ss adjusted the relative durations of the two elements of the test micropattern, a complex set of changes occurred in other stimulus parameters as well. These additional changes came about as a consequence of the interaction of a number of unavoidable instrumental, physical, and psychophysical constraints which will be discussed briefly.

In order to prevent click transients from interfering with the psychophysical matching task, the rise and decay times of the elements could not be instantaneous. The same rise and decay time $(7.0 \mathrm{msec})$ were used as were used in all previous experiments. In order to keep the duration of each micropattern 
Table 2

\begin{tabular}{|c|c|c|c|c|}
\hline \begin{tabular}{c}
\multicolumn{1}{c}{$\begin{array}{c}\text { Micropattern } \\
\text { Parameters }\end{array}$} \\
$\begin{array}{l}\text { Element Sequence } \\
\text { Element Frequency }\end{array}$
\end{tabular} & \multicolumn{2}{|c|}{$\begin{array}{l}\text { Standard Micropattern } \\
\text { (Set by E) }\end{array}$} & \multicolumn{2}{|c|}{$\begin{array}{c}\text { Test Micropattern } \\
\text { (Bold Face Values Set by S) }\end{array}$} \\
\hline $\begin{array}{l}\text { Element Sequence } \\
\text { Element Frequency } \\
\text { Element Duration } \\
\text { Energy Ratio }(\mathrm{Y}: \mathrm{X})\end{array}$ & $\begin{array}{c}\text { Element } X_{\mathrm{s}} \\
1500 \mathrm{~Hz} \\
12.0 \mathrm{msec}\end{array}$ & $\begin{array}{c}\text { Element } Y_{\mathrm{S}} \\
1300 \mathrm{~Hz} \\
12.0 \mathrm{msec}\end{array}$ & $\begin{array}{l}\text { Element } Y_{\mathrm{t}} \\
1300 \mathrm{~Hz} \\
15.27 \mathrm{msec} \\
15.24 \mathrm{msec}\end{array}$ & $\begin{array}{c}\text { Element } \mathrm{X}_{\mathrm{t}} \\
1500 \mathrm{~Hz} \\
\mathbf{8 . 7 3} \mathrm{msec} \pm \mathbf{0 . 1 3} \text { (MBS) } \\
8.76 \mathrm{msec} \pm \mathbf{0 . 3 6} \text { (MC) } \\
14.6: 1\end{array}$ \\
\hline
\end{tabular}

below the Hirsh-Sherrick threshold of $20 \mathrm{msec}$, the duration of the stimulus elements had to be brief. For the standard micropattern, this was $12 \mathrm{msec}$. Finally, the time constants of the audio gates operated in such a fashion that elements shorter than the time constant were markedly attenuated with respect to amplitude and became asymmetrical with respect to rise and decay times.

Figure 5A illustrates that the exponential rise of each element (reaching $1 / \mathrm{e}$ of the $1.0-\mathrm{V}$ signal in $5.0 \mathrm{msec}$ ) was permitted to last for only $5.0 \mathrm{msec}$. The audio gate then was instructed to close, which initiated the exponential decay of the tone burst, which required $7.0 \mathrm{msec}$ for completion. The duration of the first element was thus $12 \mathrm{msec}$. The second element was programmed to begin $5.0 \mathrm{msec}$ after the onset of the first element-that is, at the same instant the first element was beginning to decay. The rise of the second element was also terminated after $5.0 \mathrm{msec}$ and took $7.0 \mathrm{msec}$ to decay fully. Thus, both elements were of the same duration $(12 \mathrm{msec})$ and the same peak amplitude. Figure 5A also illustrates the initial parameters of the test micropattern also set by the $\mathrm{E}$. They are identical with those of the standard micropattern except for the temporal order of the two frequencies.

Figure 5B illustrates one step of the adjustment procedure made by the $\mathrm{S}$ on the test micropattern. The $\mathrm{S}$ merely altered a switch which increased the duration of the first element by $2.0 \mathrm{msec}$ and decreased the duration of the second element by the same amount. However, this change in duration had the following consequences, which can be seen in the tracing: The rise of the first element was permitted to last for $7.0 \mathrm{msec}$, thus increasing its amplitude slightly. Since the decay time was unaffected and remained at $7.0 \mathrm{msec}$, the duration of the first element was $14.0 \mathrm{msec}$. However, the rise of the second element (which was always initiated at the same moment the first element began to decay) was permitted to continue for only $3.0 \mathrm{msec}$. This diminished its peak amplitude. Since its decay time was unaffected, its duration was $10.0 \mathrm{msec}$. It will be noted that this reduction in duration of the second element, with its associated decrease in amplitude, actually resulted in a more marked reduction of its energy than would be the case if only its duration had been reduced.

Figure $5 \mathrm{C}$ illustrates the effects of an additional 1.25 -msec increase in the duration of the first element and an equal decrease in the duration of the second element of the test micropattern. The increase in duration of the first element necessarily increased its amplitude slightly (because of the exponential rise-time characteristics of the audio gate). The parallel decrease in the duration of the second element by $1.25 \mathrm{msec}$ cut short its rise time and thus markedly affected its amplitude. It is clear that a reduction of the duration of the second element to $8.75 \mathrm{msec}$ from $12.0 \mathrm{msec}$ has a marked effect on its amplitude, as well as the symmetry of rise and decay times. Figure 5C represents the actual shape of the two stimulus elements at a time when the two $S$ s reported that the two micropatterns of Fig. 5C were indistinguishable. The duration of the first element of the test micropattern is $15.25 \mathrm{msec}$, and the duration of the second element is $8.75 \mathrm{msec}$. To ascertain the ratio of energies in the two elements at the null point, the element wave forms of $\mathrm{Fig} .5 \mathrm{C}$ were digitized, squared, and integrated, using a computer of average transients. At the experimentally determined null point. the ratio of durations of the first to the second element was 1.75:1. However, the ratio of energies was 14.6:1 (see Table 2). This ratio of energies conveys a more meaningful measure of the degree to which the second element dominates the perception of a micropattern than does the ratio of durations.

\section{Experiment $V$}

Experiment $\mathrm{V}$ was intended to determine in more detail the nature of the perceptual interactions between the two elements of a micropattern. The $S$ was presented with a standard micropattern in which the EDs were both $12 \mathrm{msec}$ and of equal amplitude ( $80 \mathrm{~dB}$ SPL). The frequencies of the two elements of the standard micropattern were 1,500 and $1,300 \mathrm{~Hz}$ (see Fig. 6, Line A). A half-second later, the $S$ was presented with a test micropattern in which one of the elements (denoted by the letter $V$ ) had a different frequency from its "mate" in the standard micropattern. The $\mathrm{S}$ was required to adjust the frequency of this variable element (V) until the two micropatterns were perceptually indistinguishable. The $\mathrm{S}$ was permitted to deliver to himself as many pairs of micropatterns as he desired until he was satisfied with his adjustment. The frequency of the variable element (V) was recorded and another trial was instituted. Twenty null measures were made for each of 10 conditions, illustrated in Fig. 6, A.E and $A^{\prime}-E^{\prime}$. In Conditions $E$ and $E^{\prime}$, the $S$ simply made a frequency match for an isolated element. The results of Experiment V on the two Ss are found in Table 3, where the value of $(V)$ is the mean of 30 trials.

A comparison of Table 3 with Fig. 6 reveals that the differential limen (dl) for frequency is more than

Table 3

\begin{tabular}{|c|c|c|c|c|c|}
\hline $\begin{array}{c}\text { Con- } \\
\text { dition }\end{array}$ & $\begin{array}{l}\text { Mean } \\
\text { Fre- } \\
\text { quency } \\
\text { (V) }\end{array}$ & SD & $\begin{array}{l}\text { Con- } \\
\text { dition }\end{array}$ & $\begin{array}{l}\text { Mean } \\
\text { Fre- } \\
\text { quency } \\
\text { (V) }\end{array}$ & SD \\
\hline \multicolumn{6}{|c|}{ SM.C. } \\
\hline A & 1513 & \pm 17.6 & $\mathrm{~A}^{\prime}$ & 1320 & \pm 24.3 \\
\hline B & 1498 & \pm 9.9 & $B^{\prime}$ & 1307 & \pm 12.0 \\
\hline C & 1517 & \pm 20.8 & $C^{\prime}$ & 1312 & \pm 35.8 \\
\hline D & 1507 & \pm 8.4 & $\mathrm{D}^{\prime}$ & 1306 & \pm 13.5 \\
\hline $\mathrm{E}$ & 1519 & \pm 10.4 & $\mathrm{E}^{\prime}$ & 1304 & \pm 11.8 \\
\hline \multicolumn{6}{|c|}{ S M.B.S. } \\
\hline & 1519 & \pm 14.5 & $\mathrm{~A}^{\prime}$ & 1307 & \pm 27.6 \\
\hline B & 1501 & \pm 3.8 & $B^{\prime \prime}$ & 1304 & \pm 3.5 \\
\hline C & 1508 & \pm 17.4 & $C^{\prime}$ & 1327 & \pm 24.4 \\
\hline D & 1510 & \pm 6.2 & $D^{\prime}$ & 1301 & \pm 4.9 \\
\hline $\bar{E}$ & 1500 & \pm 5.1 & $\mathrm{E}^{\prime}$ & 1304 & \pm 2.7 \\
\hline
\end{tabular}


doubled in Conditions $A, A^{\prime}, C$, and $C^{\prime}$ for both Ss compared to their $\mathrm{dl}$ in Conditions $\mathrm{B}, \mathrm{B}^{\prime}, \mathrm{D}, \mathrm{D}^{\prime}, \mathrm{E}$, and $E^{\prime}$. There is no intra-S significant difference in the dl of these last six conditions. In contrast, in Conditions $\mathbf{A}$, $A^{\prime}, C$. and $C^{\prime}$ (where the $\mathrm{dl}$ was markedly elevated), the variable element was the first element in the test micropattern.

The increase in the $\mathrm{dl}$ for the first, but not for the second element, in the test micropattern indicates that the perceptual experience of the first element is degraded by the second but that the reverse is not the case. The $\mathrm{dl}$ of the second element (Conditions B, $\mathrm{B}^{\prime}, \mathrm{D}$, and $D^{\prime}$ ) did not differ from the dl measured with that element in isolation (Conditions $\mathrm{E}$ and $\mathrm{E}^{\prime}$ ).
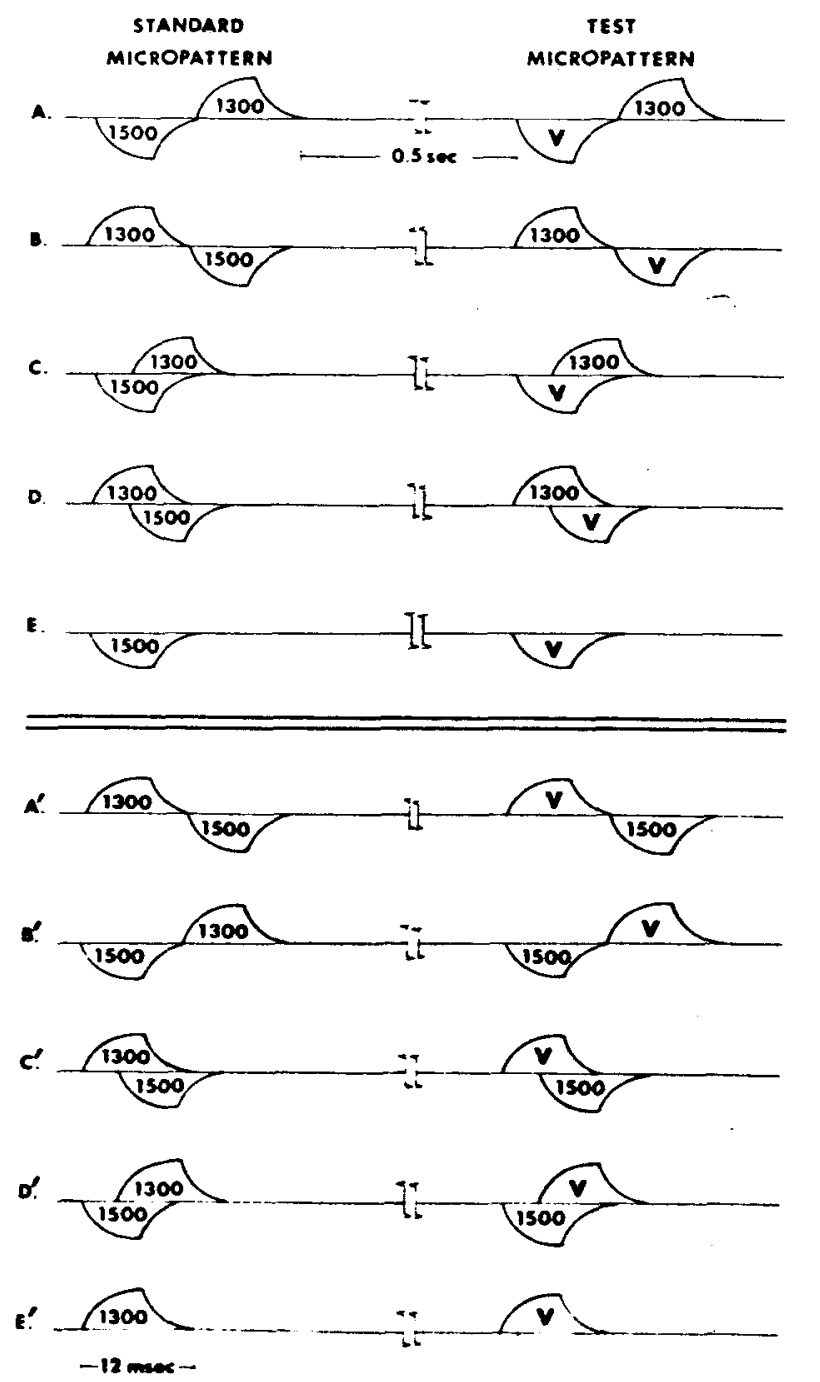

Fig. 6. Paradigm of Experiment $V$ (audition). Ten experiments, $A-E, A^{\prime}-E^{\prime}$, are illustrated. In each case, the $S$ is required to match the text micropattern to a standard micropattern by adjusting the frequency of Stimulus Element $v$ in the test micropattern. Experiments $E$ and $E^{\prime}$ are control experiments using only single elements of 1,500 and $1,300 \mathrm{~Hz}$, respectively (see Table 3 for results).
The results of Experiment $V$ indicate that the perceptual acuity of the first element (as measured by the $\mathrm{dl}$ ) is diminished. This finding, when coupled with the results of Experiment IV, suggests that the degradation of the perception of the first element involves not only an effective intensity attenuation but a despecification of its frequency as well.

\section{Vibration}

The experiments in the vibratory modality were essentially identical with those in the auditory modality. The stimulus parameters for these experiments are illustrated in the upper-left schematic of Fig. 1. The micropatterns were composed of stimulus elements having different frequencies. The frequency of Element $X$ was always $100 \mathrm{~Hz}$; Element $Y$ was 400,300 , or $150 \mathrm{~Hz}$. These values give rise to $\Delta f$ values of 300 , 200 , and $50 \mathrm{~Hz}$. The two micropatterns were presented in the same four combinations indicated in Fig. 2, and the Ss were required to report if the two micropatterns were the same or different. The element durations were $20 \mathrm{msec}$, which included a 10-msec rise and decay time. (The use of faster rise and decay times produced perceptual transients which made the experiments virtually impossible to perform.) The ON-ASYN and OFF-ASYN were both $20 \mathrm{msec}$.

The Ss reported much more difficulty in making the discriminations in the vibratory modality than they did in the auditory modality, and one of the two Ss was unable to perform at above chance levels with these short vibratory pulses. Although good performance could be achieved by this $S$ with longer element durations, the need to keep the element asynchronies unequivocally below the threshold for explicit temporal order judgments resulted in the exclusion of this $S$. The author served as the second $S$.

The pooled results on both Ss are displayed in Fig. 7 . The ordinate is the percentage of correct discriminations (out of 200 trials) and the abscissa is the $\Delta f$. As was the case in the auditory modality, a reduction in the frequency difference between the two elements degraded the performance.

Both Ss based their replies on the perceived difference in dominant frequency of vibration. As was the case in the auditory modality, the perceived dominant frequency of each micropattern was the frequency of the second element. Given the subjective difficulty in performing these judgments in the vibratory modality, further experiments such as those performed in the auditory modality were not pursued. However, the results which were obtained with these Type I alterations closely resembled those found in the auditory modality.

\section{Vision}

The experiments in the visual modality were 


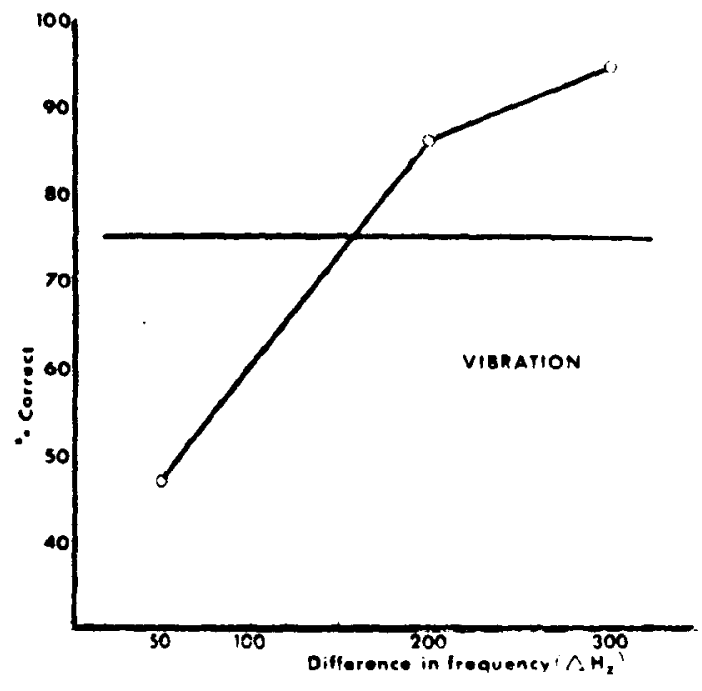

Fig. 7. Discrimination of micropatterns (vibration). Data of two Ss (see text for parametric details).

essentially identical to those previously described in audition and the vibratory sense.

In these experiments, each micropattern consisted of two elements of different wavelength $-620 \mathrm{~m} \mu$ (red) and $510 \mathrm{~m} \mu$ (green). The ED was $20 \mathrm{msec}$ for each element, and the rise and decay times were less than $0.4 \mathrm{msec}$. The Ss were required to report if the micropatterns were the same or different. The four types of temporal alterations illustrated in Fig. 1 and the same set of four combinations (Fig. 2) were used as in the auditory experiments. Identical psychophysical procedures were employed.

When exposed to a rapid sequence of these two elements, all Ss reported perceiving a yellow flash. However, they could readily discriminate between two micropatterns (in which temporal order of elements was reversed) and reported that they performed this discrimination by using a hue difference. All Ss reported that the appearance of the red-green sequence was slightly greenish-yellow, while the appearance of the green-red sequence was slightly orange-yellow.

As the element asynchrony was decreased (in Type I alterations), discriminatory performance deteriorated (see Fig. 8). The experiment was then repeated with two elements having wavelengths of 600 and $528 \mathrm{~m} \mu$. Since the results did not differ (at the 0.05 level), the data of both experiments were pooled. Figure 8 shows the pooled results for both $\Delta \lambda$ s. The ordinate is the percentage of correct discriminations. The abscissa is the element asynchrony. The experiment was attempted for the third time with elements having wavelengths of 570 and $545 \mathrm{~m} \mu$. Both Ss found it impossible to discriminate between the micropatterns and were at a chance level of performance at all values of element asynchrony.

The results of these experiments parallel those in audition and vibration (where the elements had different frequencies). With the element parameters used, the Ss perceived a yellow flash and were unable to determine the temporal order of the red and green elements. They based their judgments on the difference in hue (or lack thereof) between the two micropatterns. The dominant hue reported by the $S$ for each micropattern again correlated perfectly with the temporal order of the elements in that micropattern.

Additional studies in the visual modality were performed using wide-band colored filters. Experiments using 20 - or 10 -msec element durations passed through a Wratten No. 29 (red) and a Wratten No. 58 (green) filter showed the same dominance effect. To demonstrate that the phenomenon was not unique to the red-green component-color system, the same experiments were repeated using Wratten No.47 (blue) and Wratten No. 15 (yellow) filters. In this last case, each micropattern gave rise to a white flash. However, as expected, the sequence blue-yellow produced a yellowish-white flash and the sequence yellow-blue gave rise to a bluish-white flash-hue differences which permitted the micropatterns to be readily discriminated.

Experiments identical to Experiments IIIA and IIIB in the auditory modality were also performed in the visual modality. Once again, the element offset asynchrony proved to be the effective parameter in permitting micropattern discrimination. Type III alterations were performed with red-green as well as with blue-yellow mixtures, and gave the same results.

The adjustment experiments (Experiments IV and V)

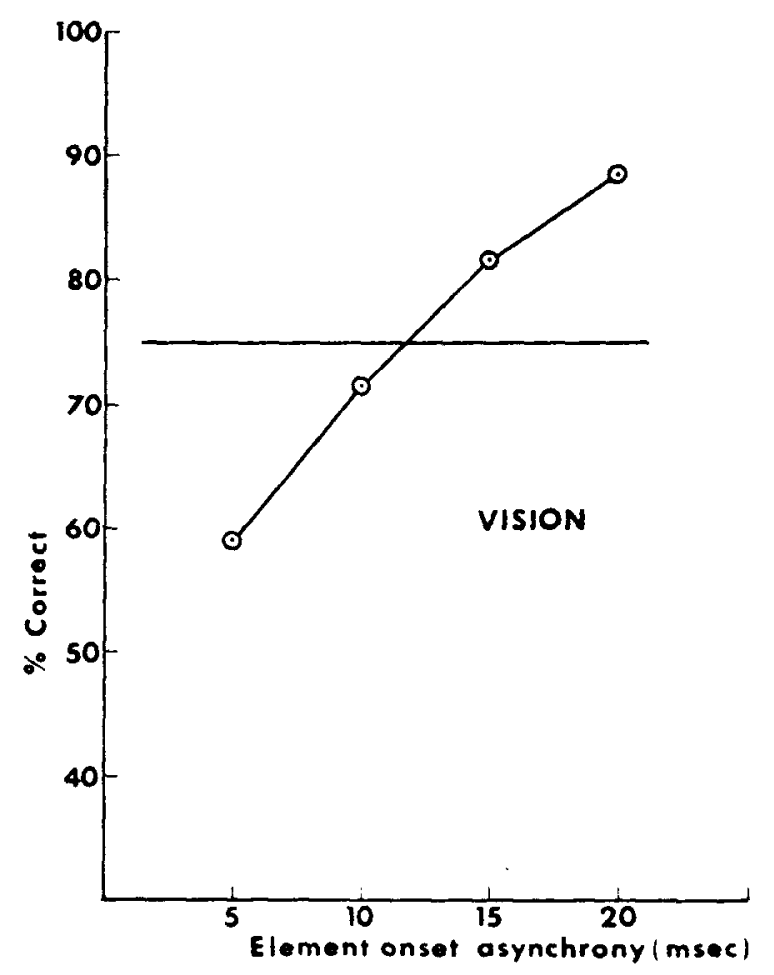

Fig. 8. Discrimination of micropatterns (vision). Data of two Ss (see text for parametric details). 
were not performed in the visual modality due to equipment limitation.

\section{DISCUSSION}

The results of these experiments demonstrate that temporal order information is conserved by perceptual systems even when the asynchrony between the stimulus elements is below the threshold for temporal order judgments. This conservation of temporal order information is achieved by a transformation into another perceptual dimension or quality-one in which there. is no subjective experience of time. This is not a new observation. It is well known, for example, that clicks delivered via earphones to the right and left ears with an interstimulus interval of a few hundred microseconds give rise to a single perceptual experience, which is lateralized (in the head) toward the ear receiving the leading stimulus. In the visual modality, the phenomenon of apparent motion is observed when two rapidly successive stimuli are presented to spatially separated retinal loci. In both cases, the interval between the two stimuli is too short to allow for any judgment of temporal order-the temporal information is conserved by means of a transformation into another (spatial) perceptual dimension. In the present experiments, the temporal information is transformed into the perceptual dimensions of "pitch" (in both the auditory and vibrotactile senses) and hue (in the visual modality).

The experimental results reveal the existence of another aspect of this process of conservation of temporal order information-that it is accomplished at the expense of a degradation of the perceptual acuity of the first of the two rapidly sequential stimuli. The degradation of the perceptual experience of the first stimulus element. with respect to its subjective intensity (Experiment IV) and with respect to its dl (Experiment V), is associated with the subjective dominance of the second element of the micropattern. In contrast. the perceptual experience of the second element appears to be unaffected by the presence of the first. The discrimination of a difference between micropatterns thus depends on a retroactive effect of the second stimulus element on the first within each micropattern. What is the nature of this retroactive effect?

The two most general concepts that deal with the perceptual degradation of the first of two rapidly successive stimuli are (a) the concept of (retroactive) masking demonstrated in all perceptual modalities and (b) the concept of metacontrast, which has been considered, heretofore, to be a property of the visual system. The relation of cach of these concepts to the present findings will be discussed in the following two sections. The third section of the discussion will be concerned with some recent experiments which might provide an alternative approach. The final section will describe some experiments in the auditory modality by other investigators which relate to the phenomenon of micropattern discrimination.

\section{Masking}

There are two aspects of the results that strongly undercut any explanation in terms of retroactive masking: The first relates to the encrgy parameters and the second to the frequency parameters of the micropattern.

In the first place, the phenomenon of retroactive masking is usually found only when the energy and/or duration of the masking stimulus is much larger than that of the target or probe stimulus. In contrast, the perceptual degradation of the first stimulus element of the micropattern is readily demonstrated with two stimulus elements of equal energy (Type I, II, and V experiments) or nearly equal energy (Type III experiments). The energy parameters of discriminable micropatterns are thus inconsistent with those which generate retroactive masking effects.

Secondly, the phenomenon of masking is most marked (in all modalities) when the masking stimulus activates the same group of receptors as the probe (first) stimulus. [In auditory theory, the concept of critical bands deals quantitatively with the observation that masking is most marked if the first stimulus is centered near the middle of the bandwidth of the masking stimulus. The amount of masking decreases markedly as the frequency of the probe stimulus is moved outside the bandwidth of the masking stimulus (Homick et al, 1969; Elliott, 1962, 1969)]. In contrast, the present experiments show unequivocally that the interaction between the two stimulus elements of each micropattern is more marked (and discrimination performance consequently improved), when the frequency difference between the elements is increased, i.e., when the critical bands of the two elements are least likely to overlap. In an extreme test of this argument, Ss were presented with micropatterns consisting of stimulus elements of 1,000 and $6,000 \mathrm{~Hz}$. Such stimuli are so far removed from each other's critical bands that retroactive masking of the first stimulus element would be negligible, the perceptual dominance of the trailing element would disappear, and the micropatterns should be nondiscriminable. This was not the case: The perceptual dominance of the trailing stimulus element was powerful, and perfect micropattern discrimination was achieved.

The same considerations pertaining to energy and wavelength parameters are applicable to the visual modality, and the results are once again inconsistent with any explanation in terms of retroactive masking.

\section{Metacontrast}

Unlike the phenomenon of masking, which is typically produced by two rapidly successive stimuli of markedly unequal energy delivered to the same receptor 
elements, the phenomenon of metacontrast is typically generated by two successive stimuli of equal energy which fall on spatially separated populations of receptors (see Lefton, 1973, for a recent review of parametric issues). In the micropattern experiments, the stimulus elements also activated two spatially separated populations of receptors. In the auditory modality, the two tone elements (even when their frequencies were not markedly different) undoubtedly stimulated spatially separate, but overlapping, populations of frequency-specific elements on the basilar membrane. In the visual modality, the two colored flashes (although falling on the same region of the retina) surely discharged overlapping populations of wavelength-specific cells. In the vibratory modality, the traveling wave in the skin resulting from two stimulus elements of different frequency probably stimulated spatially overlapping populations of receptor elements. The activation of different populations of receptors by the stimulus elements is thus analogous to the spatially separated stimuli which give rise to visual metacontrast effects.

In contrast to the similarity of spatial factors, the temporal parameters of the stimuli that give rise to metacontrast effects are quite unlike the temporal parameters of the micropatterns. In those metacontrast paradigms employing two stimuli of equal or nearly equal energy, the maximum metacontrast effect occurs when the second stimulus follows the first by a delay of approximately $100 \mathrm{msec}$. A severe attenuation and/or total loss of the metacontrast effect occurs when the interstimulus interval is appreciably shorter or longer than this value-the much-discussed U-shaped function. Although the present experiments did demonstrate a more marked perceptual interaction between the stimulus elements as the element asynchrony increased, Ss could discriminate auditory micropatterns reliably $(75 \%)$ even when the element asynchrony was only $2 \mathrm{msec}$ (see Figs. 3, 4, and Table 1). In the visual modality, the interaction effect was often detectable with element asynchronies below $5 \mathrm{msec}$. While these values are far shorter than those described in any metacontrast experiment, they are not necessarily inconsistent with such an explanation.

\section{Iconic Storage-Processing Time}

Since the work of Sperling (1963) on short-term storage effects in vision, a number of investigators have advanced a variety of models-the goal of which has been to integrate such time-dependent perceptual processes as short-term (iconic) storage, masking, metacontrast, and apparent movement (Haber \& Standing, 1969; Kahneman, 1967; Liss, 1968; Massaro, 1972; Efron, 1973). The central feature of these models is the idea that the onset of the second of two rapidly successive stimuli terminates the central processing of the information contained in the first stimulus. This premature termination of the processing of the first stimulus reduces the amount of information that can be extracted from it and thus degrades the perceptual acuity. It should be noted that these models have been generated to deal with experimental paradigms in which the two stimuli are immediately successive or follow each other with a short interstimulus interval and have not been applied to stimuli that temporally overlap.

In those micropatterns in which the two stimulus elements are consecutive (see Type I and Type II alterations of Fig. 1), these models would predict that the onset of the trailing stimulus element would arrest the central processing of the initial stimulus element. The consequent perceptual degradation of the first element in each micropattern would make the two micropatterns discriminable when the temporal order of elements is reversed. This explanation is consistent with a number of experiments performed in the visual (Haber \& Standing, 1969) and auditory (Massaro, 1972) modalities using successive stimuli without temporal overlap. For the stimuli of Experiment IIIA (see Fig. 1), the argument could possibly be extended by assuming that the onset of the second stimulus element interfered with the central processing of the initial (leading) part of the first element. This process-which would degrade only that information which makes the micropatterns distinguishable-might account for the Ss' inability to utilize the element onset asynchrony information that was characteristic of the Type IIIA experiments (see Table 1). However, it is not clear how this argument could be extended further to deal with the results of the Type IIIB experiments, where the element onsets are simultaneous and where there is no salient signal to terminate the processing of the first element. Even more difficult to explain by these models is the important effect of the ratio of element offset asynchrony to element duration, which was characteristic of the Type IIIB experiments (see Table 1).

\section{Related Phenomena in Auditory Modality}

Several recent experiments in the auditory modality which may bear a close relation to the phenomenon of micropattern discrimination warrant particular attention.

Patterson and Green (1970), using Huffman sequences (a pair of brief wave forms which differ only in phase spectra) have shown that such stimuli are discriminable. Although Ss report merely hearing two clicks, these clicks can be discriminated by virtue of a characteristic pitch difference. They concluded that "the ear is sensitive to small differences in the arrival time of energy at different frequencies and that these differences allow the observer to discriminate among the different Huffman sequences." The Patterson and Green experiment showed that temporal asynchronies as small as $1.5-2.0 \mathrm{msec}$ can be utilized by the auditory 
system-values almost identical to those obtained with micropatterns.

Nábèlek and Hirsh (1969) and Nábèlek, Nábèlek, and Hirsh (1970), using brief tone bursts of increasing or decreasing frequency, have shown that the terminal frequency of the tone burst is more significant for pitch discrimination than the initial frequency. This finding so closely resembles the phenomenon of perceptual dominance of the trailing element of micropatterns composed of two elements that it prompted further informal experiments using two micropatterns, each consisting of three 5-msec stimulus elements of different frequency. Ss could discriminate easily between two such micropatterns when the second and third element (or the first and third) had reversed temporal orders-a finding completely consistent with the Nábelek, Nábèlek, and Hirsh studies using stimuli of continuously changing frequency. A similar result was also obtained in the visual modality using micropatterns with three different hues.

The existence of virtually identical psychophysical characteristics involving micropattern discrimination in three modalities presents an unusual problem to be solved. On the one hand, an explanation is required which is sufficiently general to be equally applicable to all three modalities, which have strikingly different receptor organs, central neural connections, and functional properties. On the other hand, an explanation is required which is sufficiently specific to account quantitatively for the phenomenon in each modality (considered in isolation) in terms of its known psychophysical and neurophysiological characteristics.

\section{REFERENCES}

Broadbent, D. E. Mechanical model for human attention and immediate memory. Psychological Review, 1957, 64, 205-215.

Broadbent, D. E., \& Ladefoged, P. Auditory perception of temporal order. Journal of the Acoustical Society of America, $1959,31,1539$.

Edwards, A. E., \& Auger, R. The effect of aphasia on the perception of precedence. Procedings of the American Psychological Association, 1965, 207-208.

Efron, R. Temporal perception, aphasia and déjà vu. Brain, $1963,86,403-424$

Efron, R. An invariant characteristic of perceptual systems in the time domain. In S. Kornblum (Ed.), Attention and performance IV. New York: Academic Press, 1973.

Elliott, L. L. Backward and forward masking of probe tones of different frequency. Journal of the Acoustical Society of America, 1962, 34, 1116-1117.
Elliott, L. L. Masking of tones before, during and after brief silent periods in noise. Journal of the Acoustical Society of America, 1968, 45, 1277-1279.

Gengel, R. W., \& Hirsh, I. J. Temporal order: The effect of single versus repeated presentations, practice and verbal feedback. Perception \& Psychophysics, 1970, 7, 209-211.

Haber, R. N., \& Standing, L. G. Direct measures of short-term visual storage. Quarterly Journal of Experimental Psychology, $1969,21,43-54$.

Hirsh, I. J. Auditory perception of temporal order. Journal of the Acoustical Society of America, 1959, 31, 759-767.

Hirsh, I. J., \& Fraisse, P. Simultanéité et succession de stimuli hétérogènes. Année de Psychologie, 1964, 64, 1-19.

Hirsh, I. J., \& Sherrick, C. E. Perceived order in different sense modalities. Journal of Experimental Psychology, 1961, 62, 423-432.

Holmes, H. Disordered perception of auditory sequences in aphasia. Unpublished PhD thesis, Harvard University, 1965.

Homick, J. L., Elfner, L. F., \& Bothe, G. G. Auditory temporal masking and the perception of order. Journal of the Acoustical Society of America, 1969, 45, 712-718.

Jerger, J., Weikers, N. J., Sharbrough, F. W., \& Jerger, S. Bilateral lesions of the temporal lobe. A case study. Acta Otolaryngologica, 1969, Supplement 258.

Kahneman, D. An onset-onset law for one case of apparent motion and metacontrast. Perception \& Psychophysics, 1967, $2,577-584$.

Kristofferson, A. B. Successiveness discrimination as a two-state, quantal process. Science, 1967a, 153, 1337-1339.

Kristofferson, A. B. Attention and psychophysical time. Acta Psychologica, 1967b, 27, 93-100.

Lefton, L. A. Metacontrast: A review. Perception \& Psychophysics, 1973, 13, 161-171.

Liss, P. Does backward masking by visual noise stop stimulus processing? Perception \& Psychophysics, 1968, 4, 328-330.

Lowe, A. D., \& Campbell, R. A. Temporal discrimination in aphasoid and normal children. Journal of Speech \& Hearing Research, 1965, 8, 313-314.

Massaro, D. W. Stimulus information vs processing time in auditory pattern recognition. Perception \& Psychophysics, $1972,12,50-56$.

Nábělek, I., \& Hirsh, I. J. On the discrimination of frequency transitions. Journal of the Acoustical Society of America, $1969,45,1510-1519$.

Nábělek, I. V., Nábelek, A. K., \& Hirsh, I. J. Pitch of tone bursts of changing frequency. Journal of the Acoustical Society of America, 1970, 48, 536-553.

Patterson, J. H., \& Green, D. M. Discrimination of transient signals having identical energy spectra. Journal of the Acoustical Society of America, 1970, 48, 894-905.

Sperling, G. A model for visual memory tasks. Human Factors, $1963,5,19-31$.

Swisher, L., \& Hirsh, I. J. Brain damage and the ordering of two temporally successive stimuli. Neuropsychologia, 1972, 10, 137-152.

(Received for publication March 16, 1973; revision received June 7,1973 .) 